\title{
Diagnostics of Spectrally Resolved Transient Absorption: Surface Plasmon Resonance of Metal Nanoparticles
}

\author{
H. Ye. Seferyan, R. Zadoyan, ${ }^{\dagger}$ A. W. Wark, R. M. Corn, and V. A. Apkarian* \\ Department of Chemistry, University of California, Irvine, California 92697-2025, and Technology and \\ Applications Center, Newport Corporation, 1791 Deere Avenue, Irvine, California 92606
}

Received: August 1, 2007; In Final Form: September 21, 2007

\begin{abstract}
Time and frequency resolved transient absorption measurements yield two-dimensional images that map the dynamical correlation between the center and width of the scattering function. Global analysis of such data allows unique diagnostics of the mechanics underlying the time evolution. We specialize in the case of surface plasmon resonances of optically driven nanoparticles. We present a catalog of 2D maps that can be used to fingerprint physically meaningful cases, and we provide two experimental examples to illustrate the diagnostic value of the maps and their utility in extracting the various time constants at play. In silver nanorods, the experiment shows a $\pi / 2$ phase shift between the oscillations of the center and the width of the plasmon resonance. Inspection of the maps allows the assignment that the center of the plasmon resonance tracks the strain in shape-oscillations, while the width tracks the strain rate. This finding is the basis of the novel mechanism of plasmon damping due to electron scattering from the electrophoretic potential generated by the motion of the interfacial double layer in colloidal nanoparticles. Measurements in gold nanoparticles show over-damped oscillations, which obscure the phase correlation between the center and width of the plasmon. The damping is dominated by inhomogeneous dephasing, and the time dependence of the width, which follows the temperature of the nanoparticles, and is diagnostic of the interband transition contribution to the plasmon resonance.
\end{abstract}

\section{Introduction}

In a recent publication, we presented an analysis of spectrally resolved transient absorption in silver nanorods and demonstrated that the observed $\pi / 2$ phase shift between the modulation of the center and the width of the plasmon resonance suggests a hitherto unrecognized mechanism of plasmon broadening. ${ }^{1}$ This new mechanism is specifically related to electron scattering from the electrophoretic potential generated by the motion of interfacial double layer of colloidal nanoparticles. Here we give a more general analysis of transient absorption with all physically meaningful cases to show that the two-dimensional $(\lambda, t)$ image of the signal provides mechanistic diagnosis. Transient absorption is a general technique, and when performed with probe pulses that are much broader than the interrogated absorption feature (white light) they generate two-dimensional dynamical correlation maps between the width and the position of the resonance. The power of the method is fully realized by global fits to the images rather than separate time slices that present the dynamics at a particular wavelength. The discussion applies to transient absorption in any system where the spectral distribution is characterized by two moments, line center and line width. However, we take our example from surface plasmon resonance (SPR) spectroscopy of nanoparticles.

Optically active collective oscillation of conduction electrons in metallic nanoparticles is known as SPR. ${ }^{2}$ Optical properties of surface plasmons in metallic colloids are summarized by their characteristic extinction spectrum $\sigma(\omega, t)$ that is defined by the two moments: the center frequency and the width of the

* To whom correspondence should be addressed. E-mail: aapkaria@ uci.edu.

$\dagger$ Newport Corporation. resonance. The frequency of the plasmon resonance lies in the visible region and depends on the size and shape of the particle, the dielectric properties of the surrounding medium, ${ }^{3}$ and interparticle interactions. ${ }^{4}$ Beyond Mie theory, numerical solutions of Maxwell's equations have been employed successfully to compute the spectra of arbitrary structures. ${ }^{5}$ In recent decades, the study of plasmon resonances and vibrational modes in metallic nanoparticles has attracted a lot of attention. The interest in this area has been driven by a list of useful applications that include medical diagnostics, ${ }^{6}$ nano-optics, ${ }^{7}$ nanoelectronics, ${ }^{8-12}$ quantum dot lasers, ${ }^{13}$ nanomaterial engineering, and a myriad of applications enabled by locally enhanced electric fields and nonlinear optical phenomena mediated by them. ${ }^{14,15}$ Understanding of the effect of vibrational modes of these colloids on the heat capacity, photophysics, and elastic response was also of interest for applications in MEMS devices, ${ }^{16}$ laser-induced transformation of metals, ${ }^{17,18}$ and selective heating in condensedphase and biological systems. ${ }^{19-21}$ The fundamental interest in the subject is also driven by investigation of the scalability of the physical laws due to reduction of the size of a material that leads to drastic changes to its electronic and vibrational properties.

The ultrafast optical response of metal nanoparticles involves the excitation-relaxation dynamics of electrons, plasmons, and phonons, which is subject to spatial confinement and interfacial effects. A short-pulsed laser radiation leads to impulsive intraband excitation of conduction electrons followed by their relaxation and energy deposition into phonon modes with concomitant growth of lattice temperature and onset of coherent vibrational modes. The periodic modulation of the shape, in turn, leads to modulation of plasmon resonance peak position observed in many transient absorption experiments. ${ }^{22}$ Time and 

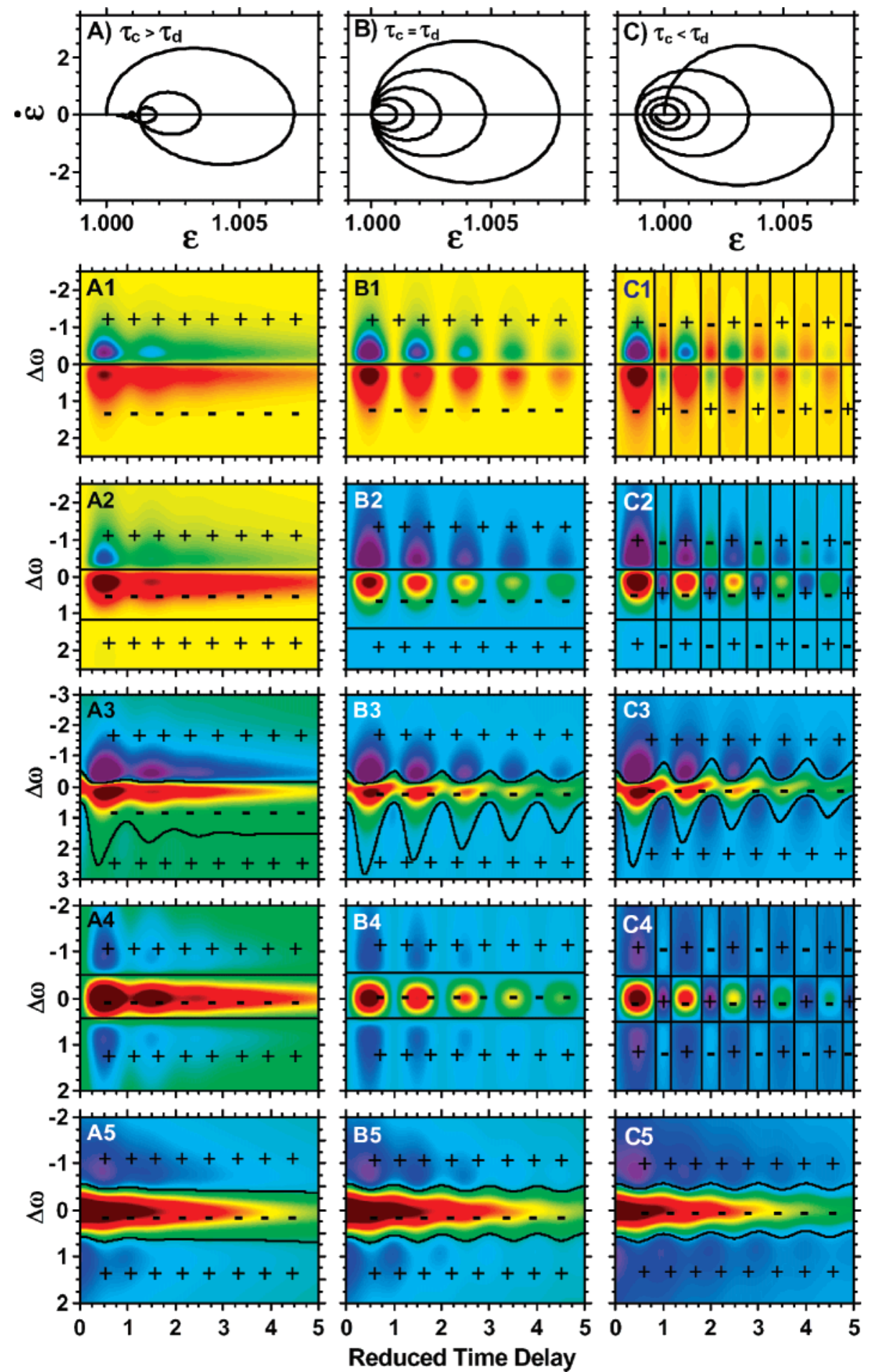

Figure 1. Catalog of 2D transient absorption maps for various mechanisms of plasmon line width and line center modulations. The top row presents the phase portrait of the forced oscillator that describes the mechanical response of the particle, sorted by the relative time constants for cooling and mechanical damping: (A) $\tau_{\mathrm{c}}>\tau_{\mathrm{d}}$, (B) $\tau_{\mathrm{c}}=\tau_{\mathrm{d}}$, and (C) $\tau_{\mathrm{c}}<\tau_{\mathrm{d}}$. The five panels in each column represent the parametrization of the line center and line width on the trajectories: (1) $\delta \omega(t) \propto \epsilon(t) ; \delta \gamma(t)=0$; (2) $\delta \omega(t) \propto \epsilon(t) ; \delta \gamma(t) \propto \epsilon(t) ;(3) \delta \omega(t) \propto \epsilon(t) ; \delta \gamma(t) \propto \dot{\epsilon}(t)$; (4) $\delta \omega(t)$ $\propto 0 ; \delta \gamma(t) \propto \epsilon(t) ;(5) \delta \omega(t) \propto \epsilon(t) ; \delta \gamma(t) \propto \Delta T(t)$. Each 2D plot of transient absorption is overlaid with the zero-level contour line, with clearly marked sign changes of the differential absorbance.

frequency resolved techniques have been used extensively to study the photodynamics in metallic colloids of different shapes. ${ }^{23-32}$ Transient absorption experiments have been carried out to extract the different time constants of electronelectron $^{33,34}$ and electron- phonon $^{35}$ relaxation time constants as well as to study the particle-to-surrounding energy transfer processes as a function of particle size, shape, and surrounding environment. ${ }^{32,36,37}$ In most of these cases, an assumption is made that the center of plasmon resonance, $\Omega$, is modulated with the mechanical shape oscillations of the particle noting that the free plasmon frequency is determined by the electron density $N / V$ that is modulated by coherent vibrations. These treatments ignore the modulation of the dielectric constant in the immediate vicinity of the particle and ignore the modulation of the width of plasmon resonance, $\Gamma$, observed and reported in many experiments without clear interpretation. 
In the present paper, we show that the zero-level contour lines in the $(\lambda, t)$ plot distinctly identify qualitative relations among various time constants that define the mechanical dynamics of coherent vibration modes. In addition, they determine the phase correlation between the center and the width of plasmon resonance and identify unique mechanisms underlying the spectral modulation. We provide a complete analysis of the $2 \mathrm{D}$ maps and discuss limiting cases. In addition, we bring two different examples of transient absorption experiments in silver and gold nanoparticles that show very different behavior.

\section{Transient Absorption Method}

Transient absorption measurements initiated with a pump pulse and probed with a time-delayed probe pulse yield the differential absorbance $\Delta A(t)=-\log \left(T_{\text {on }} / T_{\text {off }}\right)$, where $T_{\text {on }}$ and $T_{\text {off }}$ are the transmitted intensities with the pump on and off. ${ }^{1}$ Note, the change in transmission can be due to both absorption and scattering; nevertheless, the measurement is referred to as transient absorption. Spectrally resolved detection of the transmission with a probe pulse that has a significantly broader spectrum than the extinction function of the chromophore, $\sigma(\omega)$, allows a complete characterization of the time-dependent scattering function. For an extinction spectrum defined by the two moments: line-center, $\Omega_{0}$, and line-width, $\Gamma_{0}$, spectrally resolved transient absorption provides $2 \mathrm{D}$ images of the dynamical correlation between $\Omega(t)$ and $\Gamma(t)$. For a weakly scattering sample, assuming a Lorentzian line shape, the signal $\Delta A$ can be generalized in reduced variables (to within a small constant $k_{0}$ )

$$
\begin{aligned}
& \Delta A(\Delta \omega, t)= \\
& \quad k_{0}\left[\frac{1}{\Delta \omega^{2}+1 / 4}-\frac{1+\delta \gamma(t)}{(\Delta \omega+\delta \omega(t))^{2}+(1+\delta \gamma(t))^{2} / 4}\right]
\end{aligned}
$$

where $\Delta \omega \equiv\left(\omega-\Omega_{0}\right) / \Gamma_{0}$ is the reduced frequency shift relative to the original line center measured in units of the original line width; $\delta \gamma(t)=\delta \Gamma(t) / \Gamma_{0}$ and $\delta \omega(t) \equiv \delta \Omega(t) / \Gamma_{0}$ are the reduced perturbations for the width and the center of the resonance (see the Appendix). The time dependence of the perturbations $\delta \gamma(t)$ and $\delta \omega(t)$ is the principle information sought in the analysis.

\section{Physical Motivation of Limiting Behaviors}

According to Mie theory, the line center of the SPR in a metallic nanosphere is given as

$$
\Omega=\frac{\omega_{\mathrm{p}}}{\left(\epsilon_{1}+2 \epsilon_{\mathrm{m}}\right)^{1 / 2}}
$$

in which $\omega_{\mathrm{p}}$ is the free electron plasmon frequency in the metal, $\epsilon_{1}$ is the real part of the metal dielectric, and $\epsilon_{\mathrm{m}}$ is the dielectric constant of the local medium. The microscopic connection between the line center perturbation and shape oscillations of the particle is suggested by noting that because $\omega_{\mathrm{p}}=\left(n e^{2} / \epsilon_{0} m_{e}\right)^{1 / 2}$ is determined by the electron density $n=$ $N / V$ the volume oscillations of the nanoparticle should modulate the resonance frequency: $\delta \Omega / \Omega=\delta \omega_{\mathrm{p}} / \omega_{\mathrm{p}}=-\delta V /$ $2 V=(1-\epsilon) / 2$, where $\epsilon$ is the strain of the particle. In the reduced variables, the change in line center will track the stain, $\delta \omega(t)=Q(1-\epsilon(t)) / 2)$ where $Q=\Omega_{0} / \Gamma_{0}$ is the quality factor of the resonance. The time-dependent strain $\epsilon(t)$, which arises from the sudden thermal stress on irradiated particles, is obtained by treating the particle as a forced damped oscillator (see the Appendix). The large temperature jump in the immediate solvent shell of particles will lead to a change in density and therefore $\epsilon_{\mathrm{m}}$, which in turn will generate a time variation in the line center $\delta \Omega / \Omega=-\left[\epsilon_{1}+\right.$ $\left.2 \epsilon_{\mathrm{m}}(t)\right]^{-1} \delta \epsilon_{\mathrm{m}}$. This is a slower function of time, which follows the thermalization of the particle and local solvent shell back to the ambient.

The Lorentzian line shape of the plasmon resonance is based on the presumption that the bandwidth is given by the electron damping (dephasing) time. The modulation of plasmon bandwidth is then to be associated with the various electron scattering processes that can be grouped into contributions from the bulk, ${ }^{2}$ surface, ${ }^{38}$ radiative, ${ }^{14}$ and interfacial $^{2,39}$ terms:

$$
\Gamma=\gamma_{\mathrm{b}}+\gamma_{\mathrm{s}}+\gamma_{\mathrm{r}}+\gamma_{\mathrm{i}}=\gamma_{\mathrm{b}}+\frac{A v_{\mathrm{F}}}{L_{\text {eff }}}+\frac{h \kappa V}{\pi}+\gamma_{\mathrm{i}}
$$

In the size range where the dimensions of the particle become comparable to the electron scattering length in the bulk, the surface term dominates. The surface contribution can be cast in classical terms, ${ }^{38} \gamma_{\mathrm{s}}=A v_{\mathrm{F}} / L_{\mathrm{eff}}$, where $L_{\mathrm{eff}}$ is the effective scattering length that can be defined for arbitrary shapes and sizes; ${ }^{40}$ and the proportionality constant is determined experimentally as $A \sim 0.3 .^{41}$ The radiative damping contribution becomes important for the larger particles because its contribution grows with volume, $V$. The interfacial term is less specific and is used to describe scattering from the molecular structure at the metal-medium interface, especially where chemical bonding occurs. This contribution is clearly identified in the small size range of a few nanometers. ${ }^{42}$ The bulk-damping constant in turn is a sum of three different contributions from electron-electron, electron-phonon, and electron-defect scattering processes. ${ }^{43,44}$ Equation 3 suggests different mechanisms responsible for the modulation of the plasmon bandwidth. The bulk term will be modulated with the density of free electrons and hence by the volume of the nanoparticle because the increase in the density of free electrons will result in increase of the $\mathrm{e}-\mathrm{e}, \mathrm{e}-\mathrm{ph}$, and $\mathrm{e}-\mathrm{d}$ collision rates. Similarly, the second and third terms will be modulated with the volume: the electronsurface scattering term due to the change in the effective length of scattering $L_{\mathrm{eff}}$, and the radiative term due to the direct dependence on the volume of nanoparticle. The interfacial term can be modulated by the velocity of the interface, as shown in ref 1 . In addition, sudden heating of the solvent shell surrounding the particle will lead to a drop in the dielectric constant, and therefore the electrophoretic field, providing a temperaturedependent mechanism for a time-dependent line width. For excitations that involve interband transitions, as in the $\mathrm{S} \leftarrow \mathrm{D}$ transition in gold, we can expect a T-dependent line width as well.

In summary, the plasmon bandwidth can be modulated due to oscillations in volume and interfacial velocity and can have excursions that follow the temperature of the local dielectric of the medium. The relative magnitude of these effects will be different for nanoparticles of different size, shape, composition, interfacial layer, and solvent. In principle, all of the contributions with different magnitudes may coexist. Clearly, diagnostics that can identify the dominant perturbations are invaluable.

\section{2D Correlation Maps}

The mechanics of nanoparticles driven by the sudden thermal stress and their time-dependent temperature control the profile of the surface plasmon resonance. Assuming a sudden temperature jump, the trajectory of the forced oscillator can be 
classified according to the ratio between thermal cooling time, $\tau_{\mathrm{c}}$, and the mechanical damping time, $\tau_{\mathrm{d}}$. The three limiting cases are illustrated by the phase portraits of the oscillator shown in the first row of Figure 1. The distinguishing feature of case A $\tau_{\mathrm{c}}>\tau_{\mathrm{d}}$ versus $\mathrm{C} \tau_{\mathrm{c}}<\tau_{\mathrm{d}}$ is that in the latter case the oscillator shrinks below its original length as it vibrates, whereas in the former case the oscillations are around a stretched length that only returns to the original after complete cooling. The observable signals for various behaviors are then generated by the parametric dependence of $\delta \gamma(t)$ and $\delta \omega(t)$ on the strain, $\epsilon$ $(t)$, the strain rate, $\dot{\epsilon}(t)$, and temperature excursion, $\Delta T(t)$, of the particle and its immediate solvent shell. The five physically motivated cases that are considered in each column of Figure 1 are

$$
\begin{gathered}
\text { (1) } \delta \omega(t) \propto \epsilon(t) ; \delta \gamma(t)=0 \\
\text { (2) } \delta \omega(t) \propto \epsilon(t) ; \delta \gamma(t) \propto \epsilon(t) \\
\text { (3) } \delta \omega(t) \propto \epsilon(t) ; \delta \gamma(t) \propto \dot{\epsilon}(t) \\
\text { (4) } \delta \omega(t) \propto 0 ; \delta \gamma(t) \propto \epsilon(t) \\
\text { (5) } \delta \omega(t) \propto \epsilon(t) ; \delta \gamma(t) \propto \Delta T(t)
\end{gathered}
$$

The proportionality signs should be interpreted as "an explicit function of", with the functional dependences given in the Appendix. Note that the temperature profile $\Delta T(t)$ is that of an instantaneous jump followed by exponential decay.

The catalog of possible maps is presented in Figure 1. It consists of 15 image plots, with the zero-level contour lines shown as overlays. The latter, in many cases, are sufficient to diagnose the underlying mechanism of the correlation map. Straight horizontal zero-level lines imply isospectics, that is, a time invariant differential transmission $\Delta A$ at a given wavelength. To be clear, this is illustrated in the spectral cuts shown in Figure 2. Isospectics arise when only the line center is modulated, row 1 , or only the line width is modulated, row 4, or when line center and width are modulated in phase, row 2. Two isospectics arise when the line width is modulated, rows 2 and 4. This is characteristic of the Lorentzian line shape, which when broadens crosses the original spectral profile twice. The transmittance amplitude about the secondary isospectic in row 2 is smaller and may not be visible in noisy data. There are no isospectics in rows 3 and 5; however, when the motion of the oscillator is over-damped, as in column A, the modulation is small and may not be as obvious in noisy data. The absence of isospectics occurs either when the center and the width of the line are not correlated, as in row 5, or when they are out of phase, as in row 3.

The characteristic difference between the three columns can be seen in the time slices shown in Figure 3. The signal at a given wavelength is bipolar in case $\mathrm{C}$, which leads to interweaving time slices when taken on either side of the zero-contour lines. In the over-damped dynamics of case A, the time slices are mirror images that only meet at $t \rightarrow \infty$. In case $\mathrm{B}$, where the cooling time and mechanical damping times coincide, the time slices meet at $\Delta A=0$. These simply reflect the mechanics of the oscillator; the condition of whether the strain is bipolar or not as depicted in the phase portraits.

The considered cases pertain to homogeneous dynamics of a monodisperse ensemble. Dephasing due to polydispersity will lead to a Gaussian decay envelope, which can be easily incorporated as we show below.

\section{Experimental Examples}

The experiments were performed on polydisperse colloidal silver and gold nanorods in aqueous solution, prepared using
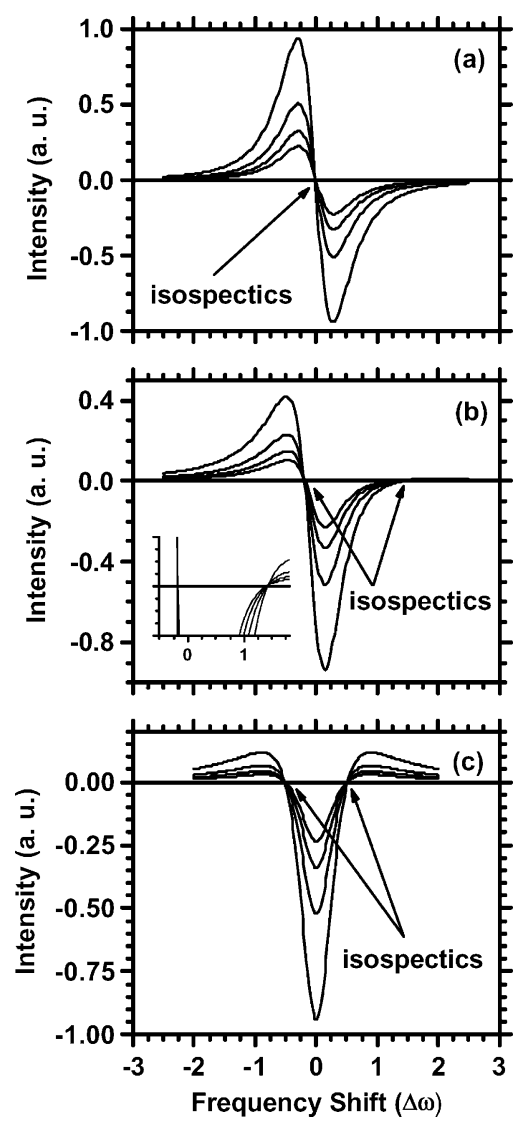

Figure 2. Spectral slices of the transient absorption signal at $\tilde{t}=0.5$, $1.5,2.5$, and 3.5 (reduced time, in units of the period of the oscillator) highlight the isospectic points for cases: (a) A1, (b) A2, and (c) A4 of Figure 1. The inset of Figure $2 \mathrm{~b}$ illustrates the double isospectics asymmetrically distributed with respect to the spectral origin; note the low transmittance amplitude past the second isospectic point.

standard chemical recipes. ${ }^{45,46}$ The measurements on the silver particles and the characterization of the sample through UVvis absorption spectra and transmission electron microscopy (TEM) were reported in ref 1 . The same approach is followed in the measurements on gold nanorods, a TEM of which is shown in Figure 4a, and the UV-vis spectrum is shown in Figure $4 \mathrm{~b}$. The Au nanorods have a mean aspect ration of $\sim 3.5$ and a standard deviation of 0.8 ; their absorption spectrum shows two spectral bands characteristic of longitudinal and transverse surface plasmon modes. The transient absorption measurements are carried out with an $800 \mathrm{~nm}$ (40 fs, $5 \mathrm{kHz}$, Spitfire, Spectra Physics) pump laser and white light probe using a commercial spectrometer (Helios, Newport). ${ }^{1}$ The samples were stirred in a $2 \mathrm{~mm}$ path length cell during the measurements.

The image plot of $\lambda-t$ resolved transient absorption in silver is presented in Figure 5a. The image can be immediately identified to belong to case $\mathrm{C} 3$ of Figure 1. The interweaving time slices of Figure 6, re-enforces the assignment. We may conclude that while the modulation of the plasmon peak follows the shape oscillations of the nanorod, the width of plasmon resonance oscillates with the strain velocity. This unique dependence identifies the spectral broadening to arise from the interfacial velocity dependent electrophoretic potential at the charged double layer of colloidal particles, as discussed in detail in ref 1 . Indeed, a satisfactory reproduction of the $2 \mathrm{D}$ image is possible, as shown in Figure 5b. The oscillation period is 75 ps, the cooling time is $65 \mathrm{ps}$, and the mechanical damping time is $150 \mathrm{ps}$. The information is sufficient to conclude that nanorods with 3:1 aspect ratio are being probed. ${ }^{1}$ Moreover, the period of oscillation shows variation with wavelength, which is used 


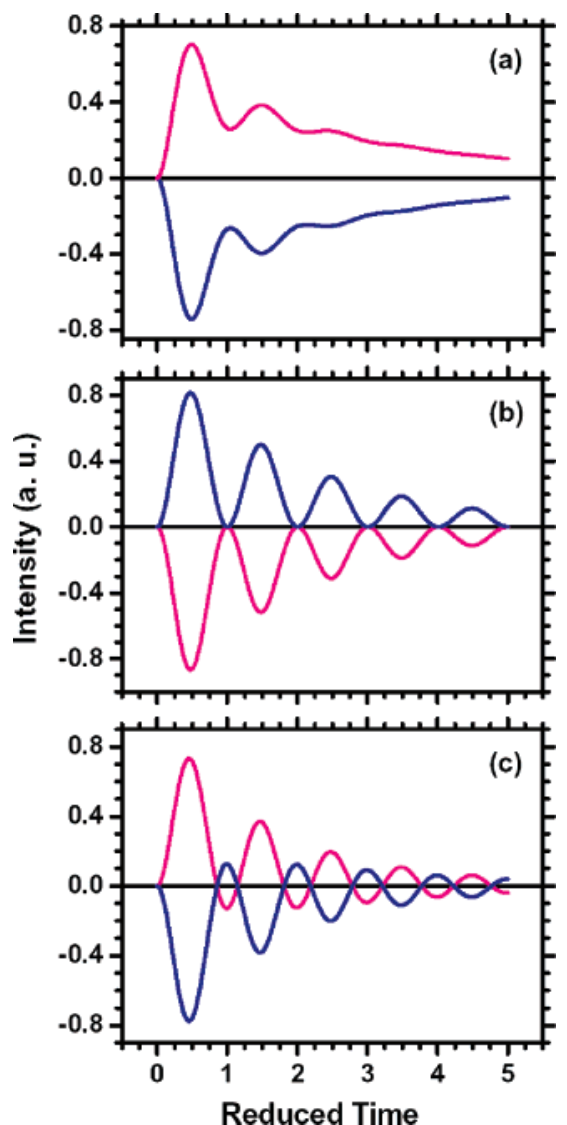

Figure 3. Two time slices taken on different sides of the isospectic, where the oscillations are anti-phase; $\mathrm{a}, \mathrm{b}$, and c correspond to cases A1, B1, and C1 of Figure 1, respectively.

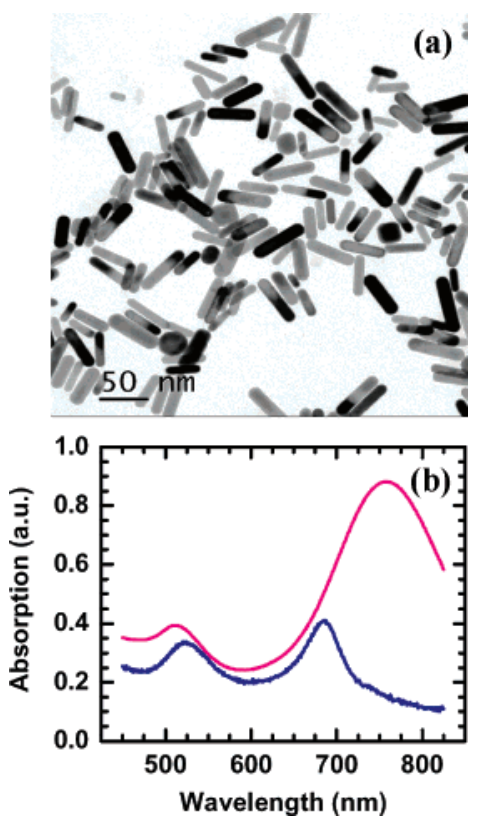

Figure 4. (a) TEM image of the $\mathrm{Au}$ nanoparticles used in the experiment. (b) Absorption of colloidal gold nanoparticles in aqueous solution. Red line: initial spectrum before exposure to $800 \mathrm{~nm}$ radiation; blue line: after exposure to radiation for $60 \mathrm{~min}$. The observed blue shift of the longitudinal band is a signature of melting and reformation of rounded nanoparticles.

to estimate that the dispersion in length of the interrogated ensemble is $6 \%$ (see ref 1 ).

The image plot of the gold nanorod sample is shown in Figure 7a. The behavior can be classified as case A5. The line center

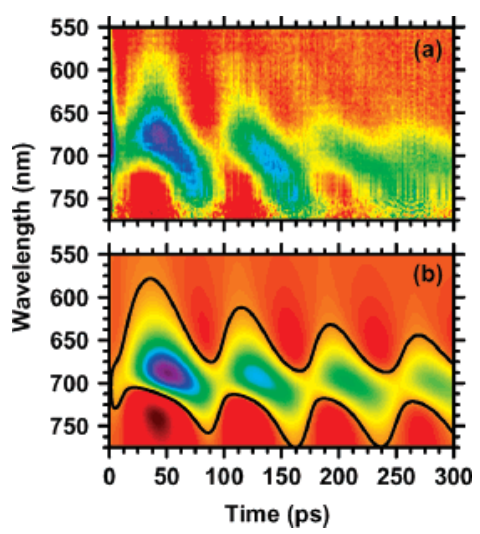

Figure 5. (a) Experimental time-frequency-resolved transient absorbance of Ag nanoparticles excited at $800 \mathrm{~nm}$. (b) Global fit according to the model introduced in the text.

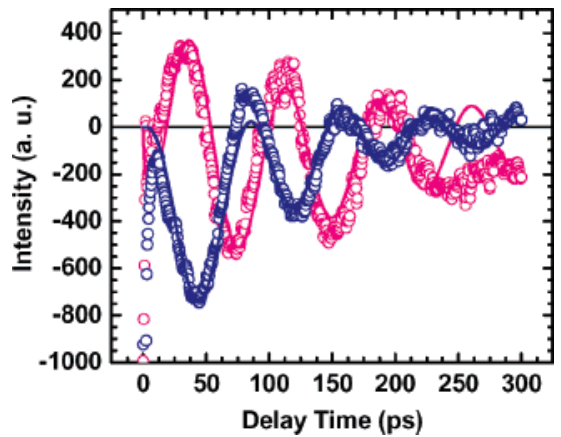

Figure 6. Time slices taken from Figure 5 at wavelengths where the oscillations are anti-phase: experiment at $725 \mathrm{~nm}$ (red circles) and 650 $\mathrm{nm}$ (blue circles), along with fits (solid lines).

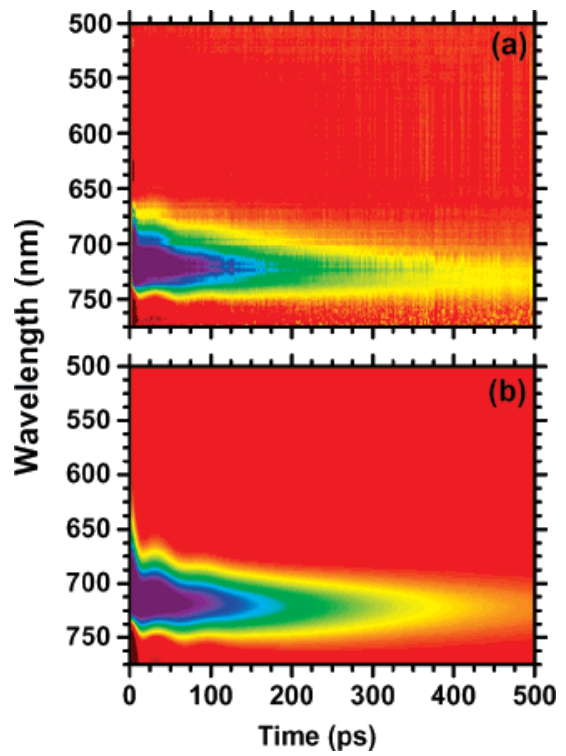

Figure 7. (a) Experimental time-frequency-resolved transient absorbance in $\mathrm{Au}$ nanorods upon excitation at $800 \mathrm{~nm}$. (b) Global fit according to the model introduced in the text.

oscillates with the strain of the nanorod, while the line width follows the temperature excursion. The fit of the $2 \mathrm{D}$ data, with explicit functional forms given in the Appendix, is shown in Figure $7 \mathrm{~b}$. The two spectral cuts shown in Figure 8 highlight the fidelity of the fit. The period of oscillation is $64 \mathrm{ps}$, which based on the bulk modulus of gold predicts a rod length of 65 $\mathrm{nm}$. The mechanical damping time constant is $45 \mathrm{ps}$, shorter than the period. The temperature excursion rises with a time constant of 1 ps and decays with a cooling time of $180 \mathrm{ps}$. 


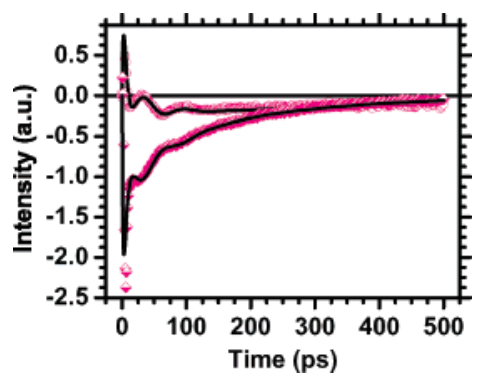

Figure 8. Two time slices taken at different sides of the plasmon resonance, where the oscillations are anti-phase, with corresponding fits according to the described model: experiment at $745 \mathrm{~nm}$ (red circles) and at $685 \mathrm{~nm}$ (red diamonds), along with fits (black lines).

Assuming a simple treatment for heat transfer, ${ }^{1}$ the cooling time suggests an aspect ratio of 1.5. Note, the TEM analysis of the pre-irradiated sample shows a distribution of aspect ratios centered at 3.5. However, upon irradiation at high intensity, the sample evolves and the longitudinal plasmon mode blue shifts, as seen in Figure 4b. The dynamical measurements were carried out on the irradiated sample at reduced laser intensity to prevent further degradation. TEM analysis of either autoclaved or lasershaped samples show reduction in the aspect ratios, which directly tracks the blue shift in absorption. The measured aspect ratio is consistent with the TEM analysis. This spectral evolution is consistent with melting and reformation of rounder particles, and has been verified through TEM analysis.

To obtain the nearly perfect fit shown in Figure 8, it is essential to assume a Gaussian decay envelope, with a time constant of $\tau_{\mathrm{deph}}(\lambda)=125-145$ ps. Evidently, the probed ensemble has significant dispersion in length. Using Hartland's analysis, ${ }^{47}$ which would strictly apply for the dephasing of the spectrally integrated transient absorption signal, dispersion in size of $11.5 \%$ would be concluded. We note that the spectrally dispersed signal shows a $\lambda$-dependent dephasing time, which would be expected for rods of different lengths (and therefore oscillation periods) to be sorted by their spectral shift. That indeed was the observation in the case of the silver rods in which the spectral variation in period was used to gauge the dispersion in length of the rods.

Note, the TEM shows much broader size dispersion in the case of silver nanoparticles than in the case of gold. However, it is the gold signal that is dominated by inhomogeneous dephasing. This is the result of selective excitation of a narrower subensemble in silver sample than in gold. In the case of silver, the $800 \mathrm{~nm}$ wavelength of the pump radiation is far from the resonance; as such, the excited subensemble is controlled by the laser bandwidth and the homogeneous spectral width of the particles. In the case of gold, the excitation is near the plasmon resonance of the bulk sample. Moreover, the homogeneous line width of $\mathrm{Au}$ plasmons is broader because of the shorter $\mathrm{e}-\mathrm{e}$ damping time. Therefore, a broader distribution of sizes is prepared in gold than in silver.

\section{Conclusions}

We presented a general treatment for the analysis of 2D time and frequency resolved transient absorption data. The treatment quite generally applies for any absorption profile that can be characterized by its two moments: center, $\Omega$, and width, $\Gamma$. We specialized our analysis to SPR bands of nanoparticles, which can have a rich variety of time-dependent spectral response depending on size, shape, preparation, interfacial structure, and local dielectric response. The key information in the $2 \mathrm{D}$ analysis is the correlation between the time dependence of the line center and line width. We considered the important set of physically motivated cases and highlighted the unique signatures that allow mechanistic assignment by a cursory inspection of the image plots. We showed that the topology of the zero-level contour line (or a suitably offset contour) and the presence of isospectics are sufficient to classify the correlation between the moments of the spectral distribution. The principles were demonstrated through two examples, with experimental data acquired in polydisperse silver and gold nanorods. In each case, the mechanism can be assigned by inspection, and refined parameters can be extracted through a more exact 2D fit of the data. The fitting procedure involves the use of trajectories of the forced, damped oscillator as the ancillary function to parametrize $\Omega(t)$ and $\Gamma(t)$.

\section{Appendix}

The extinction spectrum of the plasmon resonance, $\sigma(\omega, t)$, can be approximated as a Lorentzian that is characterized by the center, $\Omega$, and the width, $\Gamma$, of the resonance. The spectrally resolved transient absorption method measures the pulse-to pulse differential absorbance $\Delta A=-\log \left(T_{\text {on }} / T_{\text {off }}\right)$, where $T_{\text {on }}$ and $T_{\text {off }}$ are the transmitted intensities with the pump on and off. For a weakly scattering sample, the signal $\Delta A$ can be presented as the difference of the extinction spectra before and after the excitation (to within a small constant $c_{0}$ )

$$
\begin{aligned}
& \Delta A(\omega, t)= \\
& \quad c_{0}\left[\frac{\Gamma_{0}}{\left(\omega-\Omega_{0}\right)^{2}+\Gamma_{0}^{2} / 4}-\frac{\Gamma(t)}{(\omega-\Omega(t))^{2}+\Gamma(t)^{2} / 4}\right]
\end{aligned}
$$

where $\Omega(t)=\Omega_{0}+\delta \Omega(t)$ and $\Gamma(t)=\Gamma_{0}+\delta \Gamma(t)$ are the plasmon peak position and width upon perturbation by an impulsive laser radiation.

It is convenient to represent the signal $\Delta A$ in reduced variables such that the frequency is measured relative to the peak of plasmon resonance, $\Omega_{0}$, in the units of the width of the unperturbed resonance, $\Gamma_{0}$ :

$$
\begin{aligned}
& \Delta A(\omega, t)= \\
& \frac{c_{0}}{\Gamma_{0}}\left[\frac{1}{\left(\left(\omega-\Omega_{0}\right) / \Gamma_{0}\right)^{2}+1 / 4}-\right. \\
& \left.\frac{1+\delta \Gamma(t) / \Gamma_{0}}{\left(\left(\omega-\Omega_{0}\right) / \Gamma_{0}+\delta \Omega(t) / \Gamma_{0}\right)^{2}+\left(1+\delta \Gamma(t) / \Gamma_{0}\right)^{2} / 4}\right]
\end{aligned}
$$

With a new denotation $-\Delta \omega \equiv\left(\omega-\Omega_{0}\right) / \Gamma_{0}$, eq $\mathrm{A} 2$ can be written as

$$
\begin{aligned}
& \Delta A(\omega, t)= \\
& \quad \tilde{c}_{0}\left[\frac{1}{\Delta \omega^{2}+1 / 4}-\frac{1+\delta \gamma(t)}{(\Delta \omega+\delta \omega(t))^{2}+(1+\delta \gamma(t))^{2} / 4}\right]
\end{aligned}
$$

where $\tilde{c}_{0}=c_{0} / \Gamma_{0}$ is the reduced coefficient of proportionality and $\delta \gamma(t)=\delta \Gamma(t) / \Gamma_{0}$ and $\delta \omega(t) \equiv \delta \Omega(t) / \Gamma_{0}$ are the reduced perturbations for the width and the center of plasmon resonance.

The photoinduced coherent shape oscillations of the nanoparticles are treated as forced, damped oscillators

$$
\frac{d^{2} x}{d t^{2}}+\frac{2}{\tau_{\mathrm{d}}} \frac{\mathrm{d} x}{\mathrm{~d} t}+\left(\frac{2 \pi}{\tau}\right)^{2}\left(x(t)-x_{0}-\alpha \Delta T(t)\right)=0
$$

in which $\tau$ is the harmonic period of the oscillator, $\tau_{\mathrm{d}}$ is the time constant for mechanical damping, and the forcing function 
represents the thermal stress

$$
\alpha \Delta T(t)=\alpha \Delta T_{0}\left[\exp \left(-t / \tau_{\mathrm{c}}\right)-\exp \left(-t / \tau_{\mathrm{h}}\right)\right]
$$

where $\alpha$ is the thermal expansion coefficient, $\Delta T_{0}$ determines the temperature jump of the lattice due to electron-phonon scattering, and $\tau_{\mathrm{h}}$ and $\tau_{\mathrm{c}}$ are the time constants for lattice heating and cooling, respectively. In terms of the strain, $\epsilon(t)=x(t) / x_{0}$, and reduced time $\tilde{t}=t / \tau$ eq $\mathrm{A} 4$ can be written as

$$
\ddot{\epsilon}+\left(2 / \tilde{\tau}_{\mathrm{d}}\right) \dot{\epsilon}+4 \pi^{2}(\epsilon(\tilde{t})-(1+\tilde{\alpha} \Delta T(\tilde{t})))=0
$$

in which $\tilde{\tau}_{\mathrm{d}}=\tau_{\mathrm{d}} / \tau$ and $\tilde{\alpha}=\alpha / x_{0}$ are the reduced damping constant and the thermal expansion coefficient, respectively, and the differentiation is by the reduced time, $\tilde{t}$. The various limiting behaviors of the oscillator are represented by the phase portraits, which are shown in the top row of Figure 1.

The microscopic connection between the plasmon line center and the mechanical dynamics of the nanoparticle is suggested by noting that because the free plasmon frequency is determined by the electron density $n=N / V$ volume oscillations should modulate the resonance frequency: $\delta \Omega / \Omega=\delta \omega_{\mathrm{p}} / \omega_{\mathrm{p}}=-\delta V /$ $2 V$. Consequently, the reduced perturbation for the line center of resonance, $\delta \omega(t)$, is expressed as

$$
\begin{aligned}
\delta \omega(t) \equiv \delta \Omega(t) / \Gamma_{0}=- & \frac{\Omega_{0}}{\Gamma_{0}} \frac{\delta V}{2 V}=-\frac{\Omega_{0}}{\Gamma_{0}} \frac{\delta x}{2 x_{0}} \equiv- \\
& \frac{\Omega_{0}}{\Gamma_{0}} \frac{x(t)-x_{0}}{2 x_{0}}=Q(1-\epsilon(t)) / 2
\end{aligned}
$$

where $x_{0}$ and $x(t)$ are the linear dimensions of the nanoparticle before and after the excitation, $Q=\Omega_{0} / \Gamma_{0}$ is the quality factor of the resonance line shape, and $\epsilon(t)$ is the photoinduced, timedependent strain of the nanoparticle. The effect of the dielectric response of the surrounding medium is simply parametrized by the time profile of the temperature $\Delta T(\tilde{t})$, be it for the line center or the line width. The microscopic connection between the modulations of the plasmon line width and the mechanical dynamics is more diverse and can be dominated by the strain $\epsilon(t)$, the strain rate $\dot{\epsilon}(t)$, or by the temperature $\Delta T$ as discussed in Section III. Because the effect is one of increased electron scattering, the line width is not allowed to be less than the original value, $\Gamma_{0}$. Thus, taking both mechanical and thermal contributions for the case of strain rate dependence, the line width perturbation is parametrized as

$$
\delta \gamma(t)=c_{1} \dot{\epsilon}(t)+c_{2} \Delta T(t)
$$

For polydisperse ensembles, as in the case of the Au nanoparticles, the contribution of inhomogeneous dephasing can be incorporated and the signal can be well reproduced by the following functional forms for the perturbations of the plasmon linecenter, $\delta \lambda(t)$, and line width, $\delta \Gamma(t)$

$$
\begin{gathered}
\delta \lambda(t)=\frac{\lambda_{0}}{2}[\epsilon(t)-1] \exp \left(-t^{2} / \tau_{\mathrm{deph}}^{2}(\lambda)\right)+c_{1}\left[\exp \left(-t / \tau^{*}\right)-\right. \\
\left.\exp \left(-t / \tau_{\mathrm{h}}\right)\right] \text { (A9) } \\
\delta \Gamma(t)=c_{2} \Delta T(t)
\end{gathered}
$$

where $\lambda_{0}$ is the plasmon peak position in wavelength units before excitation, and $\tau_{\text {deph }}(\lambda)$, a linear function of wavelength, is the dephasing time associated with the polydispersity of the sample. The second term in eq A9 represents a fast rise and fall that accounts for the observed initial bleach and decay that is completed in $\tau^{*}=5 \mathrm{ps}$. This is presumed to be the contribution from the interband transition in gold.

Note, while the physically motivated formulations of transient absorbance are presented as a function of unitless frequency shift, $\Delta \omega$, the final plots of simulations in Figures $5 \mathrm{~b}$ and $7 \mathrm{~b}$ are made as a function of wavelength, $\lambda$, to coincide with experiments.

\section{References and Notes}

(1) Zadoyan, R.; Seferyan, H. Ye.; Wark, A. W.; Corn, R. M.; Apkarian, V. A. J. Phys. Chem. C 2007, 111, 10836.

(2) Kreibig, U.; Vollmer, M. Optical Properties of Metal Clusters; Springer: Berlin, 1995.

(3) Link, S.; El-Sayed, M. A. Int. Rev. Phys. Chem. 2007, 19, 409.

(4) Daniels, J. K.; Chumanov, G. J. Electroanal. Chem. 2005, 575, 203.

(5) Kelly, K. L.; Coronado, E.; Zhao, L. L.; Schatz, G. C. J. Phys. Chem. B 2003, 107, 668 .

(6) Mirkin, C. A.; Letsinger, R. L.; Mucic, R. C.; Storhoff, J. J. Nature 1996, 382, 607 .

(7) Quinten, M.; Leitner, A.; Krenn, J. R.; Aussenegg, F. R. Opt. Lett. 1998, 23, 1331 .

(8) Klein, D. L.; Roth, R.; Lim, A. K. L.; Alivisatos, A. P.; McEuen, P. L. Nature 1997, 389, 699.

(9) Martel, R.; Schmidt, T.; Shea, H. R.; Hertel, T.; Avouris, P. Appl. Phys. Lett. 1998, 73, 2447.

(10) Rueckes, T.; Kim, K.; Joselevich, E.; Tseng, G. Y.; Cheung, C. L.; Lieber, C. M. Science 2000, 289, 94.

(11) Black, C. T.; Murray, C. B.; Sandstrom, R. I.; Sun, S. H. Science 2000, 290, 1131

(12) Cui, Y.; Lieber, C. M. Science 2001, 291, 851.

(13) Klimov, V. I.; Mikhailovsky, A. A.; Xu, S.; Malko, A.; Hollingsworth, J. A.; Leatherdale, C. A.; Eisler, H. J.; Bawendi, M. G. Science 2000, 290, 314.

(14) Wokaun, A.; Gordon, J. P.; Liao, P. F. Phys. Rev. Lett. 1982, 48, 957.

(15) Kneipp, K.; Wang, Y.; Kneipp, H.; Perelman, L. T.; Itzkan, I.; Dasari, R.; Feld, M. S. Phys. Rev. Lett. 1997, 78, 1667.

(16) Micromechanics and Nanoscale Effects: MEMs Multi-Scale Materials and Micro-Flows; Harik, V. M., Luo, L.-S., Eds.; Kluwer Academic: Dordrecht, 2004.

(17) Kurita, H.; Takami, A.; Koda, S. Appl. Phys. Lett. 1998, 72, 789.

(18) Kamat, P. V.; Flumiani, M.; Hartland, G. V. J. Phys. Chem. B 1998, $102,3123$.

(19) Huttmann, G.; Birngruber, R. IEEE J. Select. Top. Quantum Electron. 1999, 5, 954.

(20) Lin, C. P.; Kelly, N. W.; Sibayan, S. A. B.; Latina, M. A.; Anderson, R. R. IEEE J. Select. Top. Quantum Electron. 1999, 5, 963.

(21) Hirsch, L. R.; Stafford, R. J.; Bankson, J. A.; Sershen, S. R.; Rivera, B.; Price, R. E.; Hazle, J. D.; Halas, N. J.; West, J. L. Proc. Natl. Acad. Sci. U.S.A. 2003, 100, 13549.

(22) Hartland, G. V. Annu. Rev. Phys. Chem. 2006, 57, 403.

(23) Bigot, J. Y.; Merle, J. C.; Cregut, O.; Daunois, A. Phys. Rev. Lett. 1995, 75,4702

(24) Shahbazyan, T. V.; Perakis, I. E.; Bigot, J. Y. Phys. Rev. Lett. 1998 81,3120 .

(25) Perner, M.; Gresillon, S.; Marz, J.; von Plessen, G.; Feldmann, J.; Porstendorfer, J.; Berg, K. J.; Berg, G. Phys. Rev. Lett. 2000, 85, 792.

(26) Hu, M.; Wang, X.; Hartland, G. V.; Mulvaney, P.; Juste, J. P.; Sader, J. E. J. Am. Chem. Soc. 2003, 125, 14925.

(27) Okada, N.; Hamanaka, Y.; Nakamura, A.; Pastoriza-Santos, I.; LizMarzan, L. J. Phys. Chem. 2004, 108, 8751.

(28) Huang, W. Y.; Qian, W.; El-Sayed, M. A. J. Phys. Chem. B 2005, $109,18881$.

(29) Petrova, H.; Lin, C. H.; Hu, M.; Chen, J. Y.; Siekkinen, A. R.;

Xia, Y. N.; Sader, J. E.; Hartland, G. V. Nano Lett. 2007, 7, 1059.

(30) Guillon, C.; Langot, P.; Del, Fatti, N.; Vallee, F.; Kirakosyan, A. S.; Shahbazyan, T. V.; Cardinal, T.; Treguer, M. Nano Lett. 2007, 7, 138.

(31) Hu, M.; Petrova, H.; Wang, X.; Hartland, G. V. J. Phys. Chem. B 2005, 109, 14426.

(32) Hartland, G. V. Phys. Chem. Chem. Phys. 2004, 6, 5263.

(33) Schoenlein, R. W.; Mittleman, D. M.; Shiang, J. J.; Alivisatos, A. P.; Shank, C. V. Phys. Rev. Lett. 1993, 70, 1014.

(34) Mittleman, D. M.; Schoenlein, R. W.; Shiang, J. J.; Colvin, V. I.; Alivisatos, A. P.; Shank, C. V. Phys. Rev. B 1994, 49, 14435.

(35) Park, S.; Pelton, M.; Liu, M.; Guyot-Sionnest, P.; Scherer, N. F. J. Phys. Chem. C 2007, 111, 116; and references therein.

(36) Del Fatti, N.; Voisin, C.; Chevy, F.; Valee, F.; Flytzanis, C. J. Chem. Phys. 1999, 110, 11484. 
(37) Bonacina, L.; Callegari, A.; Bonati, C.; van Mourik, F.; Chergui, M. Nano Lett. 2006, 6, 7.

(38) Kreibig, U. J. Phys. F: Metal Phys. 1974, 4, 999.

(39) Hövel, H.; Fritz, S.; Hilger, A.; Kreibig, U.; Volmer, M. Phys. Rev. $B$ 1993, 48, 18178 .

(40) Coronado, E. A.; Schatz, G. A. J. Chem. Phys. 2003, 119, 3926.

(41) Berciaud, S.; Cognet, L.; Tamarat, P.; Lounis, B. Nano Lett. 2005, 5,515 .
(42) Bauer, C.; Abid, J. P.; Fermin, D.; Girault, H. H. J. Chem. Phys. 2004, 120, 9302.

(43) Perner, M.; Bost, P.; Lemmer, U.; von Plessen, G.; Feldmann, J.; Becker, U.; Mennig, M.; Schmitt, M.; Schmidt, H. Phys. Rev. Lett. 1997, 78, 2192.

(44) Del Fatti, N.; Vallee, F. Appl. Phys. B 2001, 73, 383.

(45) Lee, P. C.; Meisel, D. J. Phys. Chem. 1982, 86, 3391.

(46) Keir, R.; Sadler, D.; Smith, W. E. Appl. Spectrosc. 2002, 56, 551

(47) Hartland, G. V. J. Chem. Phys. 2002, 116, 8048. 